\title{
Disruption of Otoferlin Alters the Mode of Exocytosis at the Mouse Inner Hair Cell Ribbon Synapse
}

\begin{abstract}
Hideki Takago 1,2,3*, Tomoko Oshima-Takago 1,2,3,4 and Tobias Moser 1,3,4,5,6
${ }^{1}$ Institute for Auditory Neuroscience and InnerEarLab, University Medical Center Göttingen, Göttingen, Germany, ${ }^{2}$ Department of Rehabilitation for Sensory Functions, Research Institute, National Rehabilitation Center for Persons with Disabilities, Saitama, Japan, ${ }^{3}$ Collaborative Research Center 889 Cellular Mechanisms of Sensory Processing, Göttingen, Germany, ${ }^{4}$ Göttingen Graduate School for Neurosciences and Molecular Biosciences, University of Göttingen, Göttingen, Germany, ${ }^{5}$ Auditory Neuroscience Group, Max Planck Institute for Experimental Medicine, Göttingen, Germany, ${ }^{6}$ Synaptic Nanophysiology Group, Max Planck Institute for Biophysical Chemistry, Göttingen, Germany
\end{abstract}

Sound encoding relies on $\mathrm{Ca}^{2+}$-mediated exocytosis at the ribbon synapse between cochlear inner hair cells (IHCs) and type I spiral ganglion neurons (SGNs). Otoferlin, a multi- $\mathrm{C}_{2}$ domain protein, is proposed to regulate $\mathrm{Ca}^{2+}$-triggered exocytosis at this synapse, but the precise mechanisms of otoferlin function remain to be elucidated. Here, performing whole-cell voltage-clamp recordings of excitatory postsynaptic currents (EPSCs) from SGNs in otoferlin mutant mice, we investigated the impact of Otof disruption at individual synapses with single release event resolution. Otof deletion decreased the spontaneous release rate and abolished the stimulus-secretion coupling. This was evident from failure of potassium-induced IHC depolarization to stimulate release and supports the proposed role of otoferlin in $\mathrm{Ca}^{2+}$ sensing for fusion.

OPEN ACCESS

Edited by:

Michael R. Kreutz,

Leibniz Institute for Neurobiology (LG),

Germany

Reviewed by:

Martin Heine,

Johannes Gutenberg University

Mainz, Germany

Kirill Volynski,

University College London,

United Kingdom

${ }^{*}$ Correspondence:

Hideki Takago

takago-hideki@rehab.go.jp

Received: 29 September 2018

Accepted: 19 December 2018

Published: 09 January 2019

Citation:

Takago H, Oshima-Takago T and Moser T (2019) Disruption of Otoferlin

Alters the Mode of Exocytosis at the Mouse Inner Hair Cell Ribbon

Synapse.

Front. Mol. Neurosci. 11:492. doi: 10.3389/fnmol.2018.00492
A missense mutation in the Otof gene (pachanga), in which otoferlin level at the $\mathrm{IHC}$ plasma membrane was lowered without changing its $\mathrm{Ca}^{2+}$ binding, also reduced the spontaneous release rate but spared the stimulus-secretion coupling. The slowed stimulated release rate supports the hypothesis that a sufficient abundance of otoferlin at the plasma membrane is crucial for the vesicle supply. Large-sized monophasic EPSCs remained present upon Otof deletion despite the drastic reduction of the rate of exocytosis. However, EPSC amplitude, on average, was modestly decreased. Moreover, a reduced contribution of multiphasic EPSC was observed in both Otof mutants. We argue that the presence of large monophasic EPSCs despite the exocytic defect upon Otof deletion supports the uniquantal hypothesis of transmitter release at the $\mathrm{IHC}$ ribbon synapse. Based upon the reduced contribution of multiphasic EPSC, we propose a role of otoferlin in regulating the mode of exocytosis in $\mathrm{HHCs}$.

Keywords: auditory, cochlea, hair cell, spiral ganglion neuron, ribbon synapse, otoferlin, calcium, EPSC

\section{INTRODUCTION}

$\mathrm{Ca}^{2+}$ influx and subsequent neurotransmitter release at inner hair cell (IHC) active zones govern sound encoding at the first auditory synapse. Excitatory postsynaptic currents (EPSCs) recorded from type I spiral ganglion neurons (SGNs) show remarkable variability in amplitude and shape. This EPSC heterogeneity led to the hypothesis of synchronized multiquantal release (MQR) at hair cell synapses (Glowatzki and Fuchs, 2002; Keen and Hudspeth, 2006; Goutman and Glowatzki, 2007; Neef et al., 2007; Li et al., 2009; Grant et al., 2010; Graydon et al., 2011; Schnee et al., 2013). 
MQR would cause monophasic (temporally compact) EPSCs when the exocytosis of vesicles comprising a MQR event is highly synchronized or multiphasic (temporally non-compact) EPSCs when synchronization of MQR is poor. However, whereas $\mathrm{Ca}^{2+}$ influx by presynaptic depolarization increases the EPSC amplitude at the frog hair cell synapse (Li et al., 2009; Graydon et al., 2011), neither changes in voltage-gated $\mathrm{Ca}^{2+}$ influx (Glowatzki and Fuchs, 2002; Grant et al., 2010) nor those in presynaptic $\mathrm{Ca}^{2+}$ buffering (Goutman and Glowatzki, 2007) affects the EPSC amplitude distribution at the rat IHC ribbon synapse. Strikingly, even when the presynaptic $\mathrm{Ca}^{2+}$ influx was abolished in mouse IHCs, EPSC size remained heterogeneous and the charge distribution of mono- and multiphasic EPSCs were unchanged (Chapochnikov et al., 2014). This, together with other experimental observations and modeling, led to the proposal that uniquantal release (UQR) is a candidate mechanism for exocytosis at the mammalian IHC ribbon synapse, whereby a combination of large clusters of postsynaptic AMPA receptors and presynaptic fusion pore dynamics would generate large and variably shaped EPSCs (Chapochnikov et al., 2014; for review, see Takago and Oshima-Takago, 2018). The UQR hypothesis of IHC exocytosis has received further support by recent studies manipulating presynaptic IHC $\mathrm{Ca}^{2+}$ influx (Huang and Moser, 2018) or directly measuring membrane fusion steps via cellattached membrane capacitance recordings from IHCs (Grabner and Moser, 2018).

Disruption of OTOF, coding for otoferlin, was identified to cause hereditary deafness DFNB9 (Yasunaga et al., 1999), while missense mutations of OTOF can lead to less profound hearing impairment (Varga et al., 2003; Marlin et al., 2010; Vogl et al., 2016; for review, see Pangršič et al., 2012; Moser and Starr, 2016). As a multi- $\mathrm{C}_{2}$ domain protein, otoferlin, in analogy to synaptotagmins, was initially proposed to serve as a $\mathrm{Ca}^{2+}$ sensor for fusion at the IHC ribbon synapse based on a functional analysis of a mouse line with a null mutation in otoferlin (Otof ${ }^{-/-}$, Roux et al., 2006) and biochemical studies (Johnson and Chapman, 2010). Moreover, a role of otoferlin in efficient vesicle priming is proposed based upon an analysis of a mouse line called pachanga that carries a missense mutation in otoferlin $\mathrm{C}_{2} \mathrm{~F}$ domain (Otof $f^{D 1767 G / D 1767 G}$ or Otof Pga/Pga, Pangršič et al., 2010). Whereas such a mutation does not affect $\mathrm{Ca}^{2+}$ binding, Otof Pga/Pga IHCs show reduced membrane-bound otoferlin level but unaltered fusion, thus suggesting that the hearing impairment arises from strongly reduced vesicle replenishment (Pangršič et al., 2010). This hypothesis is further supported by analyses of mouse lines carrying a missense mutation in otoferlin $\mathrm{C}_{2} \mathrm{C}$ domain that again lowers membrane-bound otoferlin level but unaltered fusion (Ofof ${ }^{515 T / I 515 T}$, Strenzke et al., 2016) and a null mutation in transmembrane recognition complex 40 receptor tryptophan-rich basic protein that is essential for the insertion of otoferlin into the endoplasmic reticulum in IHCs (Vogl et al., 2016). Thus, the abundance of otoferlin in IHCs is critical for maintaining the vesicle resupply to the ribbon-type active zone. Otoferlin's function in vesicle replenishment appears to involve the regulation of short filamentous tethering formation between synaptic vesicles and the active zone membrane (Vogl et al., 2015) and the facilitation of clearance of vesicular release sites from previously exocytosed membranes (exocytosisendocytosis coupling) via an interaction with the endocytic adaptor protein 2 (Duncker et al., 2013; Jung et al., 2015). On the other hand, a recent study utilizing mice with double missense mutations in otoferlin $\mathrm{C}_{2} \mathrm{C}$ domain that affect $\mathrm{Ca}^{2+}$ sensing (Otof ${ }^{D 515 A, D 517 A / D 515 A, D 517 A}$, Michalski et al., 2017) has reported that fast and sustained components of release are reduced despite unaltered otoferlin level, probably due to dysfunctional $\mathrm{Ca}^{2+}$ binding of otoferlin.

In order to further examine the otoferlin's function we performed postsynaptic voltage-clamp recordings from afferent boutons of type I SGNs in wild-type (Otof $\left.{ }^{+/+}\right)$, Otof ${ }^{P g a / P g a}$ and Otof $^{-/-}$mice and investigated exocytosis at the levels of single synapses and single release events. Analyzing the spontaneous and stimulated release rates as well as the amplitude and shape of EPSCs, we find evidence for roles of otoferlin in $\mathrm{Ca}^{2+}$-dependent fusion and replenishment of vesicles. Moreover, we propose an additional role of otoferlin in regulating the exocytic mode of IHCs to facilitate multiphasic EPSCs, potentially by controlling the vesicle fusion pore during uniquantal release at the IHC ribbon synapse.

\section{MATERIALS AND METHODS}

\section{Ethics Statement}

All experiments complied with national animal care guidelines in Germany and Japan and were approved by the University of Göttingen board for animal welfare together with the animal welfare office of the state of Lower Saxony (Germany) as well as National Rehabilitation Center for Persons with Disabilities animal experimentation committee (Japan).

\section{Animals and Preparations}

Postnatal day (P) 8-11 mice of either sex were used. Generation and general description of Otof $f^{P a / P g a}$ (Schwander et al., 2007) and Otof $^{-/-}$(Reisinger et al., 2011) mice were previously provided. As wild-type controls, C57BL6 mice (Otof ${ }^{+/+}$), which were not littermates of Otof $f^{P g a / P g a}$ or Otof ${ }^{-/}$mice, were employed. In total, 33 mice (13 Otof ${ }^{+/+}, 7$ Otof Pga/Pga and 13 Otof $^{-/-}$mice, respectively) were used in the present study. After decapitation under deep carbon dioxide inhalation anesthesia, the apical coils of organ of Corti were harvested out of the cochlea.

\section{Electrophysiology}

Whole-cell voltage-clamp recordings from postsynaptic boutons of mouse type I spiral ganglion neurons in apical coils of freshly dissected organ of Corti were performed as previously described for rats (Glowatzki and Fuchs, 2002; Rutherford et al., 2012) and for mice (Pangršič et al., 2010; Jing et al., 2013; Chapochnikov et al., 2014). The recording pipette resistance was 8-15 M $\Omega$ after pressure polishing (Goodman and Lockery, 2000). The intracellular solution contained (in mM): $150 \mathrm{CsCl}$ (or $150 \mathrm{KCl}$ in some recordings), $3.5 \mathrm{MgCl}_{2}, 0.1 \mathrm{CaCl}_{2}, 5 \mathrm{EGTA}$, 5 HEPES, and $2.5 \mathrm{Na}_{2} \mathrm{ATP}, \mathrm{pH}$ 7.2. The extracellular solution (artificial perilymph, aPL) for both dissection and recording 
contained (in mM): $5.8 \mathrm{KCl}, 155 \mathrm{NaCl}, 0.9 \mathrm{MgCl}_{2}, 1.3 \mathrm{CaCl}_{2}$, $0.7 \mathrm{NaH}_{2} \mathrm{PO}_{4}, 5.6$ D-glucose, and 10 HEPES, pH 7.4. In the high $\mathrm{K}^{+}$extracellular solution to depolarize presynaptic IHCs, 40 out of $155 \mathrm{mM} \mathrm{NaCl}$ were replaced with equimolar $\mathrm{KCl}$. In most recordings, tetrodotoxin $(1-2 \mu \mathrm{M})$ was added to block voltage-gated $\mathrm{Na}^{+}$channels. Currents were low-pass filtered at $5-10 \mathrm{kHz}$ and sampled at $20-50 \mathrm{kHz}$. EPSCs were recorded at a holding potential of $-90 \mathrm{mV}(\sim 4 \mathrm{mV}$ liquid junction potential not corrected) at room temperature $\left(21-24^{\circ} \mathrm{C}\right)$.

\section{Chemicals and Equipment}

All chemicals were purchased from Sigma-Aldrich (St. Louis, MO, United States) except for tetrodotoxin (Tocris Bioscience, Bristol, United Kingdom or Wako Pure Chemical Industries, Ltd., Osaka, Japan). The EPC-10 amplifier controlled by Patchmaster software (HEKA Elektronik, Lambrecht, Germany) as well as an upright microscope with differential interference contrast optics (Axioskop FS2, Carl Zeiss, Oberkochen, Germany or BX51WI, Olympus, Tokyo, Japan) was used.

\section{Data Analysis and Statistics}

For detection and analysis of EPSCs, MiniAnalysis software (Synaptosoft, Decatur, GA, United States) was used with a detection threshold set at 3-5 times greater than the root mean square (rms) of the baseline noise. To classify EPSCs into monoor multiphasic, the methods introduced by Grant et al. (2010) as well as Chapochnikov et al. (2014) were employed. For plotting, IGOR Pro (Wavemetrics, Lake Oswego, OR, United States), Sigmaplot (Systat Software Inc., San Jose, CA, United States) were used. The passive membrane properties such as series resistance $\left(\mathrm{R}_{\mathrm{s}}, 40 \pm 2 \mathrm{M} \Omega\right.$ for 13 Otof $+/+\mathrm{SGNs}, 41 \pm 3 \mathrm{M} \Omega$ for 7 Otof Pga/Pga SGNs, $40 \pm 2 \mathrm{M} \Omega$ for 13 Otof $^{-/}$- SGNs), membrane capacitance $\left(\mathrm{C}_{\mathrm{m}}, 1.9 \pm 0.2 \mathrm{pF}\right.$ for Otof ${ }^{+/+}, 1.9 \pm 0.1 \mathrm{pF}$ for Otof Pga/Pga, $1.8 \pm 0.2 \mathrm{pF}$ for Otof $\left.{ }^{-/}\right)$and membrane input resistance $\left(R_{m}, 498 \pm 141 \mathrm{M} \Omega\right.$ for Otof $+/+, 831 \pm 225 \mathrm{M} \Omega$ for Otof Pga/Pga, $651 \pm 106 \mathrm{M} \Omega$ for Otof $^{-/-}$) were calculated as previously described (Chapochnikov et al., 2014). Recordings with $\mathrm{R}_{\mathrm{s}}>50 \mathrm{M} \Omega$ or less than 20 EPSCs were excluded from the EPSC amplitude/charge or kinetics analysis, but included in the EPSC frequency analysis (except for recordings with less than 5 EPSCs) not to ignore very low-frequency auditory nerve fibers. Data is shown as mean \pm SEM. Statistical significance was evaluated by Student's $t$-test or one way ANOVA followed by post-hoc Tukey's test.

\section{RESULTS}

\section{Missense Mutation of Otoferlin Decreases the Rate of Spontaneous Release, Loss of Otoferlin Abolishes Stimulus-Secretion Coupling in IHCs}

EPSCs were recorded from afferent boutons of type I SGNs in P8-11 Otof $+/+$, Otof ${ }^{P g a / P g a}$ (missense mutation with reduced abundance of otoferlin) and Otof ${ }^{-1-}$ mice. At rest $(1.3 \mathrm{mM}$ $\mathrm{Ca}^{2+}$ and $5.8 \mathrm{mM} \mathrm{K}^{+}$in aPL), SGNs of all three genotypes

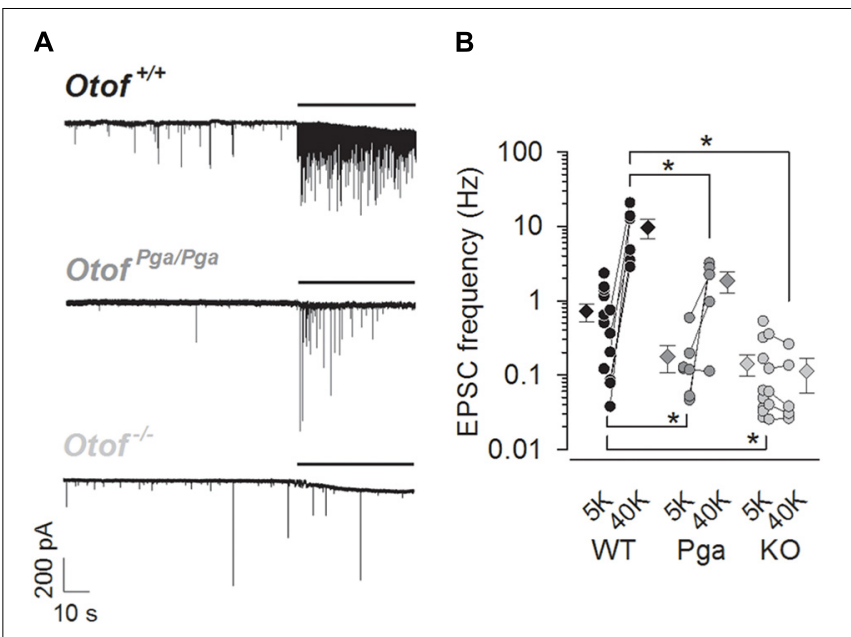

FIGURE 1 | Otoferlin regulates spontaneous and high $\mathrm{K}^{+}$-stimulated release at the mouse $\mathrm{IHC}$ ribbon synapse. (A) Sample traces of EPSCs recorded from exemplar boutons of type I SGNs from a P9 Otof $+/+$ (top), a P8 OtofPga/Pga (middle) and a P9 Otof ${ }^{-/-}$(bottom) mouse. Each recording started in the extracellular solution containing $5.8 \mathrm{mM} \mathrm{KCl}$, and $40 \mathrm{mM} \mathrm{KCl}$ was bath-applied during the time indicated by horizontal bars to depolarize presynaptic IHCs. (B) Summary of EPSC frequency in $5.8 \mathrm{mM}(5 \mathrm{~K}$, spontaneous rate) and $40 \mathrm{mM}\left(40 \mathrm{~K}\right.$, stimulated rate) $\left[\mathrm{K}^{+}\right]_{\mathrm{e}}$. Circles and diamonds indicate individual and mean \pm SEM values for Otof $+/+(W T$, black), Otof Pga/Pga (Pga, dark gray) and Otof ${ }^{-/-}$(KO, light gray) SGNs. Asterisks at the bottom (spontaneous rate) and top (stimulated rate) show significant differences $(* p<0.05)$. Note that the vertical axes for this and the next panels are shown on logarithmic scale. In some recordings, where $40 \mathrm{mM} \mathrm{KCl}$ was not applied, data points for $5.8 \mathrm{mM}\left[\mathrm{K}^{+}\right]_{\mathrm{e}}$ alone were plotted.

steadily exhibited spontaneous EPSCs (Figure 1A), but their rate was reduced in SGNs of both mutants. The frequencies of spontaneous EPSCs were $0.71 \pm 0.19 \mathrm{~Hz}$ for Otof $+/+$ $\left(n=13\right.$, Figure 1B), $0.18 \pm 0.07 \mathrm{~Hz}$ for Otof ${ }^{P g a / P g a}(n=7$, $p=0.048$ compared to Otof ${ }^{+/+}$) and $0.14 \pm 0.04 \mathrm{~Hz}$ for Otof $^{-/}$SGNs $(n=13, p=0.010$ and 0.985 compared to Otof $+/+$ and Otof $P g a / P g a$, respectively). Hence, unlike disruption of synaptotagmin I or II at the central synapses, which is thought to unclamp spontaneous release (Nishiki and Augustine, 2004; Pang et al., 2006; Xu et al., 2009; Kochubey and Schneggenburger, 2011; Lee et al., 2013; Liu et al., 2014), Otof deletion did not increase the spontaneous EPSC frequency. This argues against a clamping function of otoferlin for spontaneous release and highlights the importance of otoferlin in vesicle replenishment, since the spontaneous rate was reduced to a similar extent in Otof ${ }^{-/-}$ and Otof ${ }^{P g a / P g a}$ mice, which in a previous study showed intact vesicle fusion but impaired replenishment in IHCs (Pangršič et al., 2010).

Next, we examined the stimulus-secretion coupling in IHCs. High $\mathrm{K}^{+}$solution (40 $\mathrm{mM}$ in aPL) was bath-applied onto IHCs to increase the open probability of their Ca 1.3 L-type $\mathrm{Ca}^{2+}$ channels. The frequency of EPSCs in Otof $+/+$ SGNs was increased from $0.25 \pm 0.11(5.8 \mathrm{mM}$, control) to $9.67 \pm 2.90 \mathrm{~Hz}$ (first $10 \mathrm{~s}$ during high $\mathrm{K}^{+}$stimulation, $n=6, p=0.021$, Student's paired $t$-test, Figures $\mathbf{1 A}, \mathbf{B})$. Here, the spontaneous EPSC frequency for this control $\left(5.8 \mathrm{mM} \mathrm{K}^{+}\right)$was undervalued due 
to our preferential application of high $\mathrm{K}^{+}$-containing aPL onto low-frequency synapses, since remaining more active synapses $(1.10 \pm 0.28 \mathrm{~Hz}, n=7)$ provided a sufficient number of EPSCs for the analyses of EPSC size and shape even in the normal $\mathrm{K}^{+}$-containing aPL. In Otof $f^{P g / P g a}$ SGNs, high $\mathrm{K}^{+}$stimulation increased the frequency of EPSCs in 4 out of 5 Otof Pga/Pga SGNs from $0.20 \pm 0.10$ (control) to $1.86 \pm 0.57 \mathrm{~Hz}$ (high $\mathrm{K}^{+}$, $n=5, p=0.047$ ), indicating that the missense Otof mutation in Otof $P g a / P g a$ mice spares stimulus-secretion coupling in IHCs. The reduced rate of stimulated release in Otof ${ }^{P g a / P g a}$ IHCs $(p=0.032$ compared to Otof $+/+$ ) is compatible with impaired vesicle replenishment (Pangršič et al., 2010). In contrast, the high $\mathrm{K}^{+}$ stimulation failed to increase the EPSC frequency in Otof ${ }^{-1-}$ SGNs (control: $0.12 \pm 0.06 \mathrm{~Hz}$ vs. high $\mathrm{K}^{+}: 0.10 \pm 0.05 \mathrm{~Hz}$, $n=5, p=0.302$ ), indicating that Otof deletion abolishes stimulus-secretion coupling in IHCs. The difference in stimulated release rate between Otof ${ }^{+/+}$and Otof ${ }^{-/-}$SGNs again showed significance $(p=0.010)$.

\section{Otoferlin Disruption Decreases EPSC Amplitude and the Fraction of Multiphasic EPSCs}

We studied the effects of Otof disruption on the size and shape of EPSCs. As reported in previous studies using the organ of Corti of rats (Glowatzki and Fuchs, 2002; Grant et al., 2010), high $\mathrm{K}^{+}$stimulation of mouse Otof ${ }^{+/+}$organ of Corti increased the frequency of EPSCs without affecting the EPSC size (monophasic EPSC amplitude: $141 \pm 31 \mathrm{pA}$ for control vs. $157 \pm 24 \mathrm{pA}$ for high $\mathrm{K}^{+}$, multiphasic EPSC amplitude: $101 \pm 13 \mathrm{pA}$ for control vs. $109 \pm 16 \mathrm{pA}$ for high $\mathrm{K}^{+}$) or the fraction of multiphasic EPSCs $(41.9 \pm 3.8 \%$ for control vs. $41.1 \pm 1.7 \%$ for high $\mathrm{K}^{+}$). Moreover, EPSC size and kinetics distributions were unaltered by high $\mathrm{K}^{+}$stimulation in each phenotype SGNs (Supplementary Figures S1, S2). Therefore, EPSCs recorded in control and high $\mathrm{K}^{+}$were pooled for subsequent analyses in this study.

Surprisingly, despite the reduction in release rate and the lack of stimulus-secretion coupling, Otof ${ }^{-/}$synapses showed variable EPSC amplitudes from 11 up to several hundred pA (Figure 2). As shown in Figure 3 and Table 1, the average amplitudes of mono- and multiphasic EPSCs as well as the average charge transfer of mixed EPSCs were reduced in $\mathrm{Otof}^{-1-}$ SGNs $(p=0.013$ for monophasic EPSC amplitude, $p=0.015$ for multiphasic EPSC amplitude, $p=0.011$ for EPSC charge). There was a trend to smaller EPSC amplitudes also for Otof Pga/Pga SGNs, which, however, did not reach significance. EPSC kinetics (i.e., rise time and decay time for monophasic as well as time to peak and half width for multiphasic), on the other hand, was not different among those three groups (Figures 2, 4).

Notably, the scatter plot of EPSC amplitude versus charge shows the predominance of monophasic EPSCs clustering around the unity line in SGNs of both Otof mutants, while a substantial group of multiphasic EPSCs with smaller amplitude but similar charge exists in Otof $+/+$ SGNs (Figure 5A). The fraction of multiphasic EPSC in normal $\mathrm{K}^{+}$-containing aPL was significantly reduced in SGNs of both mutants $(41.3 \pm 2.6 \%$

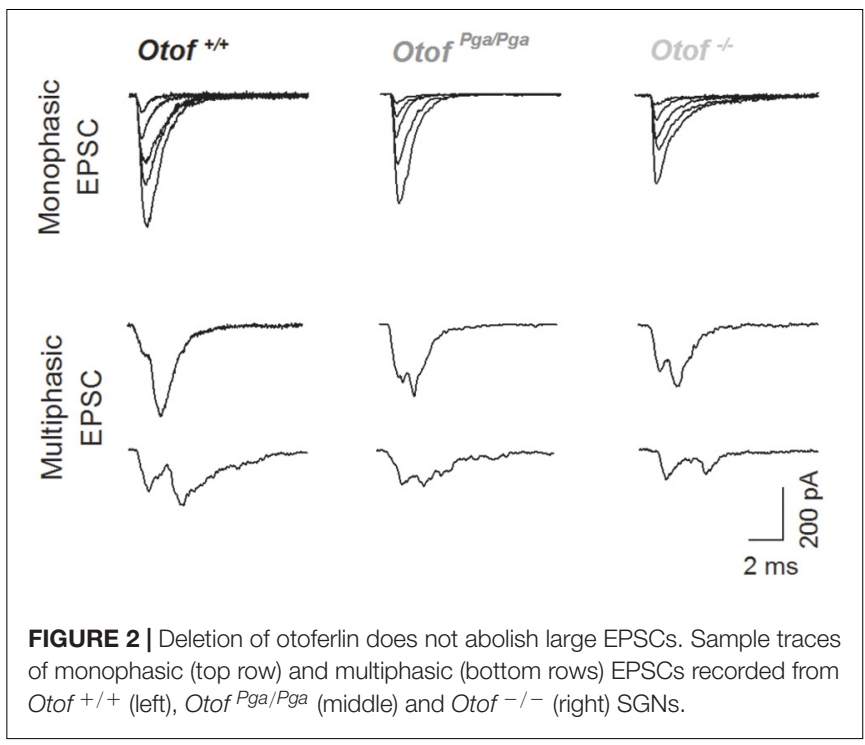

for Otof $+/+, 24.0 \pm 5.2 \%$ for Otof $P g a / P g a$ and $13.2 \pm 3.5 \%$ for Otof $^{-/-}, p<0.01$ between Otof ${ }^{+/+}$and Otof ${ }^{P g a / P g a}, p<0.001$ between Otof $+/+$ and Otof ${ }^{-/}, p=0.145$ between Otof $f^{P g a / P g a}$ and Otof ${ }^{-/-}$, Figure 5B). Also, the fraction of multiphasic EPSC in high $\mathrm{K}^{+}$-containing aPL was also significantly reduced in both mutants $\left(40.3 \pm 2.3 \%\right.$ for Otof ${ }^{+/}+, 18.7 \pm 2.9 \%$ for Otof Pga/Pga and $11.8 \pm 5.9 \%$ for Otof $f^{-/-}, p<0.01$ between Otof ${ }^{+/+}$and Otof $f^{P g a / P g a}, p<0.01$ between Otof $+/+$ and Otof ${ }^{-/-}, p=0.408$ between Otof $P g a / P g a$ and Otof ${ }^{-/-}$). Thus, monophasic EPSCs dominate transmission in the absence of otoferlin, suggesting that otoferlin regulates the mode of spontaneous and stimulated release at the first auditory synapse.

\section{DISCUSSION}

\section{Roles of Otoferlin for Stimulus-Secretion Coupling and Vesicle Replenishment}

In the present study, we recorded EPSCs from the postsynaptic afferent boutons of type I SGNs in wild-type and otoferlin mutant mice, and tested the function of otoferlin in exocytosis at the IHC ribbon synapse. We found a complete disruption of stimulussecretion coupling in Otof ${ }^{-/-}$SGNs. As $\mathrm{Ca}^{2+}$ influx and vesicle availability on a morphological level are maintained at the AZs of Otof $^{-/-}$IHCs (Roux et al., 2006), we argue that $\mathrm{Ca}^{2+}$ no longer efficiently drives fusion in the absence of otoferlin. This supports the role of otoferlin as a $\mathrm{Ca}^{2+}$ sensor of fusion (Roux et al., 2006; Johnson and Chapman, 2010; Michalski et al., 2017). But how is otoferlin-independent spontaneous release regulated? Possible mechanisms include (1) an additional high affinity $\mathrm{Ca}^{2+}$ sensor whose capacity is saturated at resting $\left[\mathrm{Ca}^{2+}\right]_{\mathrm{i}}$ and (2) a $\mathrm{Ca}^{2+}$ independent release process. For (1), the activation range of the remaining $\mathrm{Ca}^{2+}$ sensor should be below the resting $\left[\mathrm{Ca}^{2+}\right]_{\mathrm{i}}$ of a few tens of nanomolar (Beutner and Moser, 2001). However, to date, there has been no report about such a molecule that meets this requirement. The closest candidate $\mathrm{Ca}^{2+}$ sensor for fusion is 


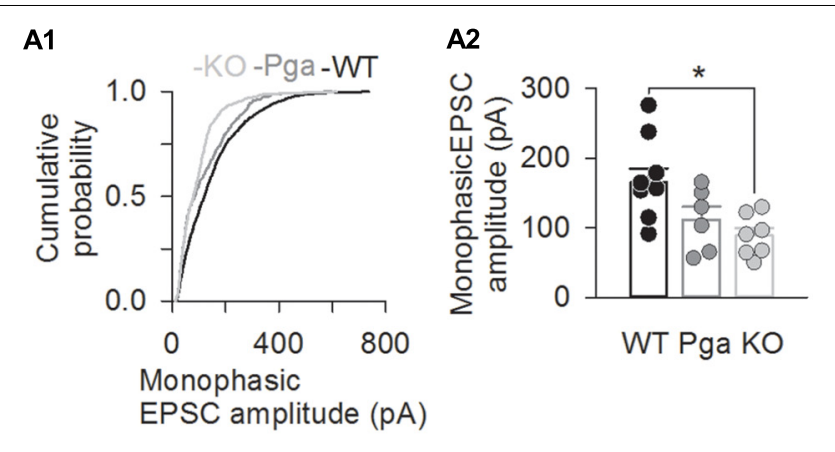

B1
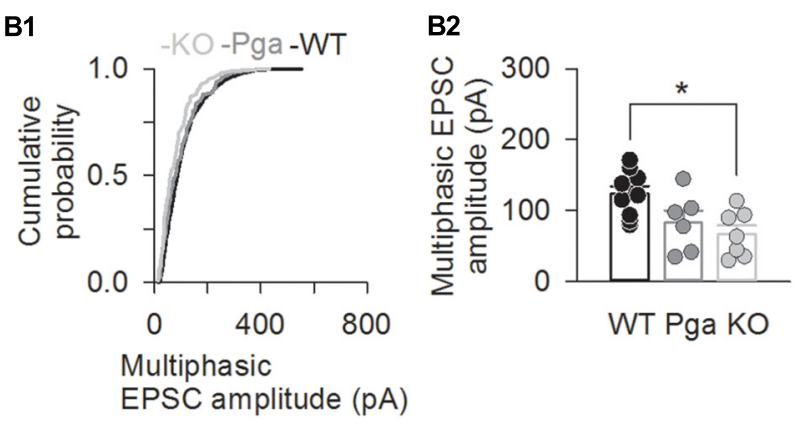

C1

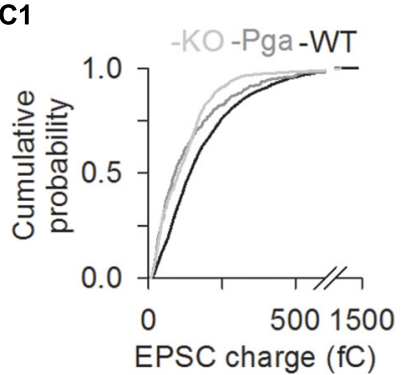

C2

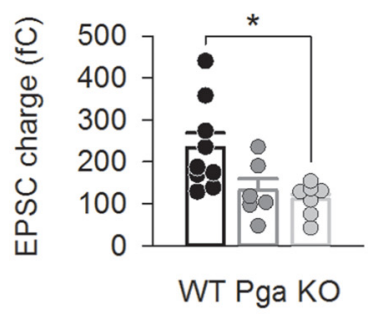

FIGURE 3 | Otoferlin deletion decreases EPSC amplitude and charge. (A-C) EPSC size for Otof ${ }^{+/+}$(black), Otof Pga/Pga (dark gray) and Otof $-1-$ (light gray) SGNs. Significant differences between Otof $+/+$ and Otof $-1-$ SGNs $\left({ }^{*} p<0.05\right)$. Cumulative histograms of monophasic EPSC amplitude (A1), multiphasic EPSC amplitude (B1) and mono- and multiphasic (mixed) EPSC charge (C1) as well as individual (circles) and mean \pm SEM (bars) values of monophasic EPSC amplitude (A2), multiphasic EPSC amplitude (B2) and mono- and multiphasic EPSC charge (C2) are shown. Note that a break line is inserted in the horizontal axis of panel C2 to clearly demonstrate the differences among groups.

the $\mathrm{C}_{2}$ domain protein Doc2b that promotes membrane fusion at submicromolar $\mathrm{Ca}^{2+}$ concentrations (but higher than $100 \mathrm{nM}$ ) (Groffen et al., 2010).

Interestingly, the absence of otoferlin did not increase the spontaneous EPSC frequency, which is in contrast to the effect of ablation of synaptotagmin I (Xu et al., 2009) or II (Pang et al., 2006) in the mouse central neurons, further highlighting differences among otoferlin and synaptotagmin I (Reisinger et al., 2011). We note here that the "spontaneous" release from resting IHCs likely includes $\mathrm{Ca}^{2+}$-evoked release that is triggered by rare openings of
TABLE 1 | EPSC size in SGN afferent boutons of wild-type and Otof mutant mice.

\begin{tabular}{lccc}
\hline & $\begin{array}{c}\text { Monophasic EPSC } \\
\text { amplitude (pA) }\end{array}$ & $\begin{array}{c}\text { Multiphasic EPSC } \\
\text { amplitude (pA) }\end{array}$ & $\begin{array}{c}\text { EPSC charge } \\
\text { transfer (fC) }\end{array}$ \\
\hline Otof $^{+/+}$ & $166 \pm 20$ & $123 \pm 11$ & $234 \pm 35$ \\
$(n=9)$ & $(1473$ EPSCs $)$ & $(1049$ EPSCs $)$ & $(2522$ EPSCs) \\
Otof Pga/Pga & $112 \pm 18$ & $83 \pm 17$ & $132 \pm 28$ \\
$(n=6)$ & $(301$ EPSCs $)$ & $(100$ EPSCs $)$ & $(401$ EPSCs $)$ \\
Otof $-/-$ & $89 \pm 11$ & $67 \pm 12$ & $103 \pm 13$ \\
$(n=7)$ & $(479$ EPSCs $)$ & $(101$ EPSCs $)$ & $(580$ EPSCs $)$ \\
\hline
\end{tabular}

Cav1.3 channels. We consider it likely that the reduction of spontaneous release reflects a combination of disrupted $\mathrm{Ca}^{2+}$-triggered spontaneous release of IHCs and reduced vesicle replenishment.

Previous studies showed that otoferlin abundance at the IHC plasma membrane correlates with the presynaptic function and sound encoding (Strenzke et al., 2016). Otof Pga/Pga mice, wherein otoferlin membrane abundance is attenuated down to $3 \%$ of Otof $+/+$ mice (Pangršič et al., 2010; Strenzke et al., 2016), displays lower EPSC frequency upon high $\mathrm{K}^{+}$-induced IHC depolarization. Although our combination of postsynaptic recordings and high $\mathrm{K}^{+}$stimulation cannot track fast stimulussecretion coupling, the robust EPSC rates in Otof $\mathrm{Pga} / \mathrm{Pga}$ afferents (Figure 1A) suggests that $\mathrm{Ca}^{2+}$-triggered membrane fusion is intact despite the mutation in the $\mathrm{C}_{2} \mathrm{~F}$ domain (i.e., D1767G, Pangršič et al., 2010). On the other hand, the dual mutations in the $\mathrm{C}_{2} \mathrm{C}$ domain (i.e., D515A and D517A), which alter its $\mathrm{Ca}^{2+}$-binding (Johnson and Chapman, 2010) but preserves otoferlin level (Michalski et al., 2017), impairs both vesicle fusion and replenishment functions of otoferlin, suggesting that $\mathrm{Ca}^{2+}$-sensing of otoferlin is critical for both steps of exocytosis (Michalski et al., 2017). Thus, not only $\mathrm{Ca}^{2+}$-sensing capacity but also plasma membrane level of otoferlin is essential for IHC exocytosis.

\section{Multiquantal Versus Uniquantal Release: Can Otoferlin Disruption Provide Insight?}

The heterogeneity of EPSC amplitude and shape is a hallmark of ribbon synapses, but the underlying mechanisms are not well understood. Notably, the mammalian IHC ribbon synapse differs from other ribbon synapses such as the amphibian and reptile hair cell synapses (Li et al., 2009; Graydon et al., 2011; Schnee et al., 2013) as well as the mammalian retinal bipolar-AII cell synapse (Singer et al., 2004; Mehta et al., 2013), where low $\left[\mathrm{Ca}^{2+}\right]_{\mathrm{i}}$ conditions break EPSCs down into unitary events. In contrast, at the rodent IHC ribbon synapse the EPSC amplitude remains sizable despite the massive buffering of presynaptic $\mathrm{Ca}^{2+}$ (Goutman and Glowatzki, 2007) or abolition of $\mathrm{Ca}^{2+}$ influx (Chapochnikov et al., 2014). This and other findings have led us to consider an alternative hypothesis for explaining EPSC amplitude and shape heterogeneity at the IHC synapse: the large EPSC amplitude may reflect activation of a large number of postsynaptic AMPA receptors (Saito, 1990; Meyer et al., 2009) activated by glutamate liberated from a single synaptic vesicle. Post-fusion regulation of such uniquantal release 

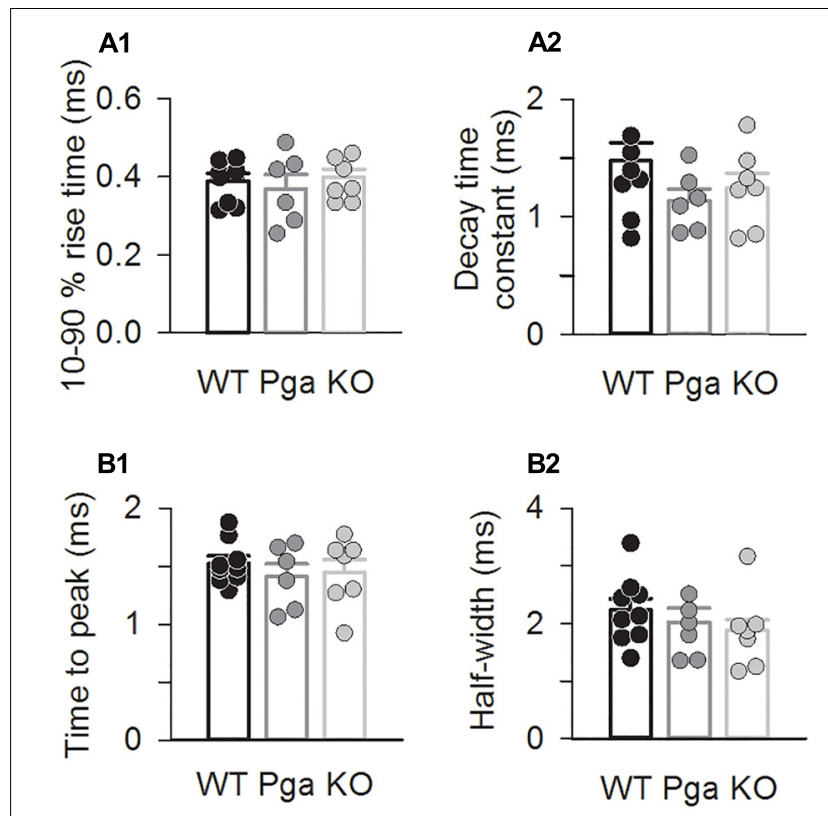

FIGURE 4 | Otoferlin disruption does not change EPSC kinetics (A,B) EPSC kinetics for Otof $+/+$ (black), Otof Pga/Pga (dark gray) and Otof ${ }^{-1-}$ (light gray) SGNs. Individual (circles) and mean \pm SEM (bars) values of 10-90\% rise time (A1) and decay time constant (A2) of monophasic EPSCs as well as time to peak (B1) and half-width (B2) of multiphasic EPSCs. No significant differences.

by a dynamic fusion pore may explain multiphasic EPSCs (successive bouts of release through a flickering pore) and small monophasic EPSCs (incomplete release of a vesicle's glutamate content) (Chapochnikov et al., 2014; Huang and Moser, 2018; for review, see Moser and Vogl, 2016; Takago and Oshima-Takago, 2018).

Given the presence of large monophasic EPSCs at the Otof ${ }^{-1-}$ IHC afferent synapse (Figure 2), i.e., under conditions of strongly reduced rate of release that seem not permissive for synchronized fusion of multiple vesicles, we favor the interpretation that uniquantal release prevails in IHCs. Interestingly, the fraction of multiphasic EPSCs was smaller when otoferlin was disrupted. Within the framework of the uniquantal release hypothesis of IHC exocytosis this can be explained as favoring full-collapse fusion and/or singular fusion pore openings. Accordingly, otoferlin would then enhance promote fusion pore flickering. Alternatively, within the framework of multiquantal release hypothesis, which the present study cannot exclude, our observation might be explained as highly synchronized fusion of a smaller number of vesicles upon Otof disruption. Besides, either hypothetic mechanism might be modulated by altered $\mathrm{Ca}^{2+}$ signaling at the release site as might have resulted from the scaffolding function of Otoferlin for $\mathrm{Ca}^{2+}$ channels (Hams et al., 2017) or attenuated fast-inactivating $\mathrm{Ca}^{2+}$ currents in Otoferlin-lacking IHCs (Vincent et al., 2017). However, we note that we had previously found normal $\mathrm{Ca}^{2+}$ signals at the individual IHC AZs in Otof ${ }^{P g a / P g a}$ mice (Pangršič et al., 2010). Further testing these hypotheses and elucidating the

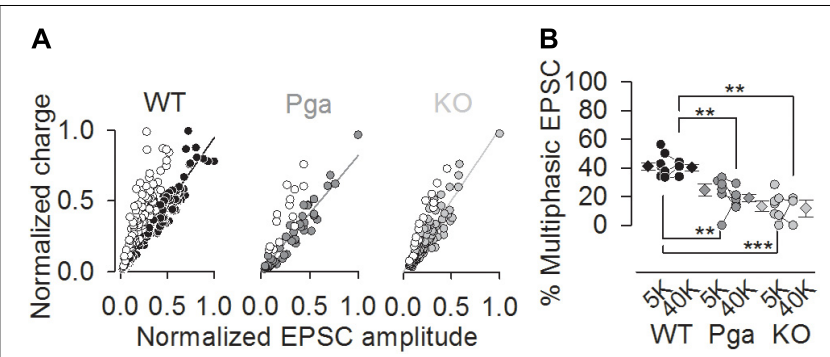

FIGURE 5 | Otoferlin disruption decreases the fraction of multiphasic EPSCs (A,B) Exocytic mode of IHC exocytosis for Otof $+/+$ (black), Otof Pga/Pga (dark gray) and Otof $-1-$ (light gray) SGNs. (A) Scatter plot of amplitude versus charge of monophasic (filled circles) and multiphasic (open circles) EPSCs derived from exemplar Otof $+/+$, Otof Pga/Pga and Otof $-/-$ SGNs. Note that the Otof Pga/Pga and Otof -/- EPSCs cluster predominantly around the regression lines for monophasic EPSCs, which is indicative of less multiphasic EPSCs in Otof mutants than in Otof $+/+$. (B) Summary of the EPSC mode in $5.8 \mathrm{mM}\left(5 \mathrm{~K}\right.$, spontaneous rate) and $40 \mathrm{mM}\left(40 \mathrm{~K}\right.$, stimulated rate) $\left[\mathrm{K}^{+}\right]_{\mathrm{e}}$. Circles and diamonds indicate individual and mean \pm SEM values of the percentage of multiphasic EPSCs for Otof $+/+$ (WT, black), Otof Pga/Pga (Pga, dark gray) and Otof $-1-$ (KO, light gray) SGNs. Asterisks at the bottom (spontaneous rate) and top (stimulated rate) show significant differences $(* * p<0.01$ and $* * * p<0.001)$.

underlying molecular events remain important goals for future studies.

A smaller fraction of multiphasic EPSCs was also found in Otof $P g a / P g a$ SGNs (Figure 5) and we suggest that the reduction of otoferlin levels at the plasma membrane suffices to alter the IHC exocytic mode. Tens of missense mutations have been described for patients with otoferlin-related deafness (for review, see Pangršič et al., 2012; Santarelli et al., 2015). It is noteworthy that even a missense mutation of otoferlin such as D1767G (pachanga) drastically decrease the heterogeneity of EPSC shape (this study). Although the functional significance of multiphasic EPSCs at the IHC ribbon synapse remains to be clarified, a wide range of spike jitter in the SGN afferent boutons caused by variable EPSP waveforms (Rutherford et al., 2012) may contribute to the heterogeneity of auditory nerve fiber responses in response to sound (Liberman, 1982; Grant et al., 2010). By promoting the shift in IHC exocytic mode, otoferlin may serve to endow the diversity in sound encoding at this ribbon-type synapse.

\section{AUTHOR CONTRIBUTIONS}

HT and TM designed the study. HT and TO-T performed experiments and analyzed the data. HT, TO-T, and TM wrote the manuscript. The experiments were performed at the University Medical Center Göttingen and Research Institute of National Rehabilitation Center for Persons with Disabilities.

\section{FUNDING}

This study was supported by a fellowship of MED-EL company (to HT) as well as grants from the German Research Foundation 
through the Collaborative Research Center 889 (Project A2 to TM) and JSPS KAKENHI (Grant Nos. 25670722 and 16K11204 to HT).

\section{ACKNOWLEDGMENTS}

We would like to thank Dr. Ulrich Müller and Dr. Ellen Reisinger for providing otoferlin mutant mice as well as Dr. Elisabeth Glowatzki and Dr. Darina Khimich for teaching postsynaptic patch clamp technique. We also would like to thank Drs. Masao Tachibana, Tomoyuki Takahashi, Takeshi Sakaba, Jeong-Seop Rhee, Nikolai Chapochnikov, Chao-Hua Huang, Yukihiro Nakamura, Yoshinori Sahara, Shinichi Iwasaki, Kensuke Watanabe, Ken Kitamura, Yuko Seko, and Koichi Mori for comments and helps.

\section{REFERENCES}

Beutner, D., and Moser, T. (2001). The presynaptic function of mouse cochlear inner hair cells during development of hearing. J. Neurosci. 21, 4593-4599. doi: 10.1523/JNEUROSCI.21-13-04593.2001

Chapochnikov, N. M., Takago, H., Huang, C. H., Pangršič, T., Khimich, D., Neef, J., et al. (2014). Uniquantal release through a dynamic fusion pore is a candidate mechanism of hair cell exocytosis. Neuron 83, 1389-1403. doi: 10.1016/j.neuron.2014.08.003

Duncker, S. V., Franz, C., Kuhn, S., Schulte, U., Campanelli, D., Brandt, N., et al. (2013). Otoferlin couples to clathrin-mediated endocytosis in mature cochlear inner hair cells. J. Neurosci. 33, 9508-9519. doi: 10.1523/JNEUROSCI.5689-12. 2013

Glowatzki, E., and Fuchs, P. A. (2002). Transmitter release at the hair cell ribbon synapse. Nat. Neurosci. 5, 147-154. doi: 10.1038/nn796

Goodman, M. B., and Lockery, S. R. (2000). Pressure polishing: a method for reshaping patch pipettes during fire polishing. J. Neurosci. Methods 100, 13-15. doi: 10.1016/S0165-0270(00)00224-7

Goutman, J. D., and Glowatzki, E. (2007). Time course and calcium dependence of transmitter release at a single ribbon synapse. Proc. Natl. Acad. Sci. U S A. 104, 16341-16346. doi: 10.1073/pnas.0705756104

Grabner, C. P., and Moser, T. (2018). Individual synaptic vesicles mediate stimulated exocytosis from cochlear inner hair cells. Proc. Natl. Acad. Sci. U S A. 115, 12811-12816. doi: 10.1073/pnas.1811814115

Grant, L., Yi, E., and Glowatzki, E. (2010). Two modes of release shape the postsynaptic response at the inner hair cell ribbon synapse. J. Neurosci. 30, 4210-4220. doi: 10.1523/JNEUROSCI.4439-09.2010

Graydon, C. W., Cho, S., Li, G. L., Kachar, B., and von Gersdorff, H. (2011). Sharp $\mathrm{Ca}^{2+}$ nanodomains beneath the ribbon promote highly synchronous multivesicular release at hair cell synapses. J. Neurosci. 31, 16637-16650. doi: 10.1523/JNEUROSCI.1866-11.2011

Groffen, A. J., Martens, S., Díez Arazola, R., Cornelisse, L. N., Lozovaya, N., de Jong, A. P. et al. (2010). Doc2b is a high-affinity $\mathrm{Ca}^{2+}$ sensor for spontaneous neurotransmitter release. Science 327, 1614-1618. doi: 10.1126/science.1183765

Hams, N., Padmanarayana, M., Qiu, W., and Johnson, C. P. (2017). Otoferlin is a multivalent calcium-sensitive scaffold linking SNAREs and calcium channels. Proc. Natl. Acad. Sci. U S A 114, 8023-8028. doi: 10.1073/pnas.1703240114

Huang, C. H., and Moser, T. (2018). $\mathrm{Ca}^{2+}$ regulates the kinetics of synaptic vesicle fusion at the afferent inner hair cell synapse. Front. Cell. Neurosci. 12:364. doi: 10.3389/fncel.2018.00364

Jing, Z., Rutherford, M. A., Takago, H., Frank, T., Fejtova, A., Khimich, D., et al. (2013). Disruption of the presynaptic cytomatrix protein bassoon degrades ribbon anchorage, multiquantal release, and sound encoding at the hair cell afferent synapse. J. Neurosci. 33, 4456-4467. doi: 10.1523/JNEUROSCI.349112.2013

\section{SUPPLEMENTARY MATERIAL}

The Supplementary Material for this article can be found online at: https://www.frontiersin.org/articles/10.3389/fnmol. 2018.00492/full\#supplementary-material

FIGURE S1 | High $\mathrm{K}^{+}$stimulation does not alter the EPSC size distribution in wild-type or Otoferlin mutant SGNs (A-C) Cummulative histograms of monophasic EPSC amplitude (A), multiphasic EPSC amplitude (B) and EPSC charge transfer (C) derived from exemplar Otof $+/+$, Otof Pga/Pga and Otof ${ }^{-1-}$ SGNs before $\left(5.8 \mathrm{mM}\left[\mathrm{K}^{+}\right]_{\mathrm{e}}\right.$, dotted lines) and during high $\mathrm{K}^{+}\left(40 \mathrm{mM}\left[\mathrm{K}^{+}\right]_{\mathrm{e}}\right.$, solid lines) stimulation. No significant differences in each phenotype.

FIGURE S2 | High $\mathrm{K}^{+}$stimulation does not alter the EPSC kinetics distribution in wild-type or Otoferlin mutant SGNs (A,B) Cummulative histograms of 10-90\% rise time (A1) and dacay time constant (A2) of monophasic EPSCs as well as time to rise (B1) and half-width (B2) of multiphasic EPSCs derived from exemplar Otof $+/+$, OtofPga/Pga and Otof $-/-$ SGNs before (5.8 $\left.\mathrm{mM}^{-\mathrm{K}^{+}}\right]_{\mathrm{e}}$, dotted lines) and during high $\mathrm{K}^{+}\left(40 \mathrm{mM}\left[\mathrm{K}^{+}\right]_{e}\right.$, solid lines) stimulation. No significant differences in each phenotype.

Johnson, C. P., and Chapman, E. R. (2010). Otoferlin is a calcium sensor that directly regulates SNARE-mediated membrane fusion. J. Cell. Biol. 191, 187197. doi: $10.1083 /$ jcb.201002089

Jung, S., Maritzen, T., Wichmann, C., Jing, Z., Neef, A., Revelo, N. H. et al. (2015). Disruption of adaptor protein $2 \mu(\mathrm{AP}-2 \mu)$ in cochlear hair cells impairs vesicle reloading of synaptic release sites and hearing. EMBO J. 34, 2686-2702. doi: 10.15252/embj.201591885

Keen, E. C., and Hudspeth, A. J. (2006). Transfer characteristics of the hair cell's afferent synapse. Proc. Natl. Acad. Sci. U S A. 103, 5537-5542. doi: 10.1073/ pnas.0601103103

Kochubey, O., and Schneggenburger, R. (2011). Synaptotagmin increases the dynamic range of synapses by driving $\mathrm{Ca}^{2+}$-evoked release and by clamping a near-linear remaining $\mathrm{Ca}^{2+}$ sensor. Neuron 69, 736-748. doi: 10.1016/j.tins. 2011.02 .006

Lee, J., Guan, Z., Akbergenova, Y., and Littleton, J. T. (2013). Genetic analysis of synaptotagmin C2 domain specificity in regulating spontaneous and evoked neurotransmitter release. J. Neurosci. 33, 187-200. doi: 10.1523/JNEUROSCI. 3214-12.2013

Li, G. L., Keen, E., Andor-Ardó, D., Hudspeth, A. J., and von Gersdorff, H. (2009). The unitary event underlying multiquantal EPSCs at a hair cell's ribbon synapse. J. Neurosci. 29, 7558-7568. doi: 10.1523/JNEUROSCI.0514-09.2009

Liberman, M. C. (1982). Single-neuron labeling in the cat auditory nerve. Science 216, 1239-1241.

Liu, H., Bai, H., Xue, R., Takahashi, H., Edwardson, J. M., and Chapman, E. R. (2014). Linker mutations reveal the complexity of synaptotagmin 1 action during synaptic transmission. Nat. Neurosci. 17, 670-677. doi: 10.1038/ nn. 3681

Marlin, S., Feldmann, D., Nguyen, Y., Rouillon, I., Loundon, N., Jonard, L. et al. (2010). Temperature-sensitive auditory neuropathy associated with an otoferlin mutation: deafening fever! Biochem. Biophys. Res. Commun. 394, 737-742. doi: 10.1016/j.bbrc.2010.03.062

Mehta, B., Snellman, J., Chen, S., Li, W., and Zenisek, D. (2013). Synaptic ribbons influence the size and frequency of miniature-like evoked postsynaptic currents. Neuron. 77, 516-527. doi: 10.1016/j.neuron.2012.11.024

Meyer, A. C., Frank, T., Khimich, D., Hoch, G., Riedel, D., Chapochnikov, N. M., et al. (2009). Tuning of synapse number, structure and function in the cochlea. Nat. Neurosci. 12, 444-453. doi: 10.1038/nn.2293

Michalski, N., Goutman, J. D., Auclair, S. M., Boutet de Monvel, J., Tertrais, M., Emptoz, A., et al. (2017). Otoferlin acts as a $\mathrm{Ca}^{2+}$ sensor for vesicle fusion and vesicle pool replenishment at auditory hair cell ribbon synapses. eLife 6:e31013. doi: $10.7554 /$ eLife.31013

Moser, T., and Starr, A. (2016). Auditory neuropathy-neural and synaptic mechanisms. Nat. Rev. Neurol. 12, 135-149. doi: 10.1038/nrneurol.2016.10

Moser, T., and Vogl, C. (2016). New insights into cochlear sound encoding. F1000Res. 5:F1000. doi: 10.12688/f1000research.8924.1 
Neef, A., Khimich, D., Pirih, P., Riedel, D., Wolf, F., and Moser, T. (2007). Probing the mechanism of exocytosis at the hair cell ribbon synapse. J. Neurosci. 27, 12933-12944. doi: 10.1523/JNEUROSCI.1996-07.2007

Nishiki, T., and Augustine, G. J. (2004). Synaptotagmin I synchronizes transmitter release in mouse hippocampal neurons. J. Neurosci. 24, 6127-6132. doi: 10. 1523/JNEUROSCI.1563-04.2004

Pang, Z. P., Sun, J., Rizo, J., Maximov, A., and Südhof, T. C. (2006). Genetic analysis of synaptotagmin 2 in spontaneous and $\mathrm{Ca}^{2+}$-triggered neurotransmitter release. EMBO J. 25, 2039-2050. doi: 10.1038/sj.emboj.7601103

Pangršič, T., Lasarow, L., Reuter, K., Takago, H., Schwander, M., Riedel, D., et al. (2010). Hearing requires otoferlin-dependent efficient replenishment of synaptic vesicles in hair cells. Nat. Neurosci. 13, 869-876. doi: 10.1038/nn.2578

Pangršič, T., Reisinger, E., and Moser, T. (2012). Otoferlin: a multi- $\mathrm{C}_{2}$ domain protein essential for hearing. Trends Neurosci. 35, 671-680. doi: 10.1016/j.tins. 2012.08.002

Reisinger, E., Bresee, C., Neef, J., Nair, R., Reuter, K., Bulankina, A., et al. (2011). Probing the functional equivalence of otoferlin and synaptotagmin 1 in exocytosis. J. Neurosci. 31, 4886-4895. doi: 10.1523/JNEUROSCI.5122-10.2011

Roux, I., Safieddine, S., Nouvian, R., Grati, M., Simmler, M. C., Bahloul, A., et al. (2006). Otoferlin, defective in a human deafness form, is essential for exocytosis at the auditory ribbon synapse. Cell 127, 277-289. doi: 10.1016/j.cell.2006. 08.040

Rutherford, M. A., Chapochnikov, N. M., and Moser, T. (2012). Spike encoding of neurotransmitter release timing by spiral ganglion neurons of the cochlea. J. Neurosci. 32, 4773-4789. doi: 10.1523/JNEUROSCI.4511-11.2012

Saito, K. (1990). Freeze-fracture organization of hair cell synapses in the sensory epithelium of guinea pig organ of Corti. J. Electron Microsc. Tech. 15, 173-186. doi: 10.1002/jemt.1060150209

Santarelli, R., del Castillo, I., Cama, E., Scimemi, P., and Starr, A. (2015). Audibility, speech perception and processing of temporal cues in ribbon synaptic disorders due to OTOF mutations. Hear. Res. 330, 200-212. doi: 10.1016/j.heares.2015.07. 007

Schnee, M. E., Castellano-Muñoz, M., and Ricci, A. J. (2013). Response properties from turtle auditory hair cell afferent fibers suggest spike generation is driven by synchronized release both between and within synapses. J. Neurophysiol. 110, 204-220. doi: 10.1152/jn.00121.2013

Schwander, M., Sczaniecka, A., Grillet, N., Bailey, J. S., Avenarius, M., Najmabadi, H., et al. (2007). A forward genetics screen in mice identifies recessive deafness traits and reveals that pejvakin is essential for outer hair cell function. J. Neurosci. 27, 2163-2175. doi: 10.1523/JNEUROSCI.4975-06.2007

Singer, J. H., Lassova, L., Vardi, N., and Diamond, J. S. (2004). Coordinated multivesicular release at a mammalian ribbon synapse. Nat. Neurosci. 7, 826833. doi: $10.1038 / \mathrm{nn} 1280$
Strenzke, N., Chakrabarti, R., Al-Moyed, H., Müller, A., Hoch, G., Pangrsic, T. et al. (2016). Hair cell synaptic dysfunction, auditory fatigue and thermal sensitivity in otoferlin Ile515Thr mutants. EMBO J. 35, 2519-2535. doi: 10.15252/embj. 201694564

Takago, H., and Oshima-Takago, T. (2018). Pre- and postsynaptic ionotropic glutamate receptors in the auditory system of mammals. Hear. Res. 362, 1-13. doi: 10.1016/j.heares.2018.02.007

Varga, R., Kelley, P. M., Keats, B. J., Starr, A., Leal, S. M., Cohn, E., and Kimberling, W. J. (2003). Non-syndromic recessive auditory neuropathy is the result of mutations in the otoferlin (OTOF) gene. J. Med. Genet. 40, 45-50. doi: 10.1136/ jmg.40.1.45

Vincent, P. F., Bouleau, Y., Charpentier, G., Emptoz, A., Safieddine, S., Petit, C. et al. (2017). Different $\mathrm{Ca}_{V} 1.3$ channel isoforms control distinct components of the synaptic vesicle cycle in auditory inner hair cells. J. Neurosci. 37, 2960-2975. doi: 10.1523/JNEUROSCI.2374-16.2017

Vogl, C., Cooper, B. H., Neef, J., Wojcik, S. M., Reim, K., Reisinger, E., et al. (2015). Unconventional molecular regulation of synaptic vesicle replenishment in cochlear inner hair cells. J. Cell Sci. 128, 638-644. doi: 10.1242/jcs.16 2099

Vogl, C., Panou, I., Yamanbaeva, G., Wichmann, C., Mangosing, S. J., Vilardi, F., et al. (2016). Tryptophan-rich basic protein (WRB) mediates insertion of the tail-anchored protein otoferlin and is required for hair cell exocytosis and hearing. EMBO J. 35, 2536-2552. doi: 10.15252/embj.2015 93565

Xu, J., Pang, Z. P., Shin, O. H., and Südhof, T. C. (2009). Synaptotagmin-1 functions as a $\mathrm{Ca}^{2+}$ sensor for spontaneous release. Nat. Neurosci. 12, 759-766. doi: 10.1038/nn.2320

Yasunaga, S., Grati, M., Cohen-Salmon, M., El-Amraoui, A., Mustapha, M., Salem, N., et al. (1999). A mutation in OTOF, encoding otoferlin, a FER-1like protein, causes DFNB9, a nonsyndromic form of deafness. Nat. Genet. 21, 363-369. doi: 10.1038/7693

Conflict of Interest Statement: The authors declare that the research was conducted in the absence of any commercial or financial relationships that could be construed as a potential conflict of interest.

Copyright (C) 2019 Takago, Oshima-Takago and Moser. This is an open-access article distributed under the terms of the Creative Commons Attribution License (CC BY). The use, distribution or reproduction in other forums is permitted, provided the original author(s) and the copyright owner(s) are credited and that the original publication in this journal is cited, in accordance with accepted academic practice. No use, distribution or reproduction is permitted which does not comply with these terms. 